\title{
Epidural Stimulation Induced Modulation of Spinal Locomotor Networks in Adult Spinal Rats
}

\author{
Igor Lavrov, ${ }^{1}$ Christine J. Dy, ${ }^{1}$ Andy J. Fong, ${ }^{4}$ Yury Gerasimenko, ${ }^{1,5}$ Grégoire Courtine, ${ }^{6}$ Hui Zhong, ${ }^{1}$ Roland R. Roy, ${ }^{1,3}$ \\ and V. Reggie Edgerton ${ }^{1,2,3}$ \\ ${ }^{1}$ Departments of Physiological Science and ${ }^{2}$ Neurobiology, and ${ }^{3}$ Brain Research Institute, University of California, Los Angeles, Los Angeles, California \\ 90095, ${ }^{4}$ Bioengineering Option, California Institute of Technology, Pasadena, California 91125, ${ }^{5}$ Pavlov Institute of Physiology, St. Petersburg 199034, \\ Russia, and ${ }^{6}$ University of Zurich, CH-8006 Zurich, Switzerland
}

The importance of the in vivo dynamic nature of the circuitries within the spinal cord that generate locomotion is becoming increasingly evident. We examined the characteristics of hindlimb EMG activity evoked in response to epidural stimulation at the S1 spinal cord segment in complete midthoracic spinal cord-transected rats at different stages of postlesion recovery. A progressive and phasedependent modulation of monosynaptic (middle) and long-latency (late) stimulation-evoked EMG responses was observed throughout the step cycle. During the first 3 weeks after injury, the amplitude of the middle response was potentiated during the EMG bursts, whereas after 4 weeks, both the middle and late responses were phase-dependently modulated. The middle- and late-response magnitudes were closely linked to the amplitude and duration of the EMG bursts during locomotion facilitated by epidural stimulation. The optimum stimulation frequency that maintained consistent activity of the long-latency responses ranged from 40 to $60 \mathrm{~Hz}$, whereas the shortlatency responses were consistent from 5 to $130 \mathrm{~Hz}$. These data demonstrate that both middle and late evoked potentials within a motor pool are strictly gated during in vivo bipedal stepping as a function of the general excitability of the motor pool and, thus, as a function of the phase of the step cycle. These data demonstrate that spinal cord epidural stimulation can facilitate locomotion in a time-dependent manner after lesion. The long-latency responses to epidural stimulation are correlated with the recovery of weight-bearing bipedal locomotion and may reflect activation of interneuronal central pattern-generating circuits.

Key words: CPG; spinal cord stimulation; locomotion; spinal cord; spinal cord injury; synaptic plasticity

\section{Introduction}

When the lumbosacral region of the spinal cord is isolated from supraspinal control in vivo, locomotor patterns in the hindlimbs can be induced or facilitated via pharmacological interventions and/or epidural stimulation (ES) (Barbeau and Rossignol, 1991; Iwahara et al., 1991; Chau et al., 1998; Dimitrijevic et al., 1998; Gerasimenko et al., 2003; Ichiyama et al., 2005). Although this phenomenon has been investigated extensively, the specific spinal networks responsible for generating locomotion cannot be completely described by classical models (Graham-Brown, 1911; Engberg and Lundberg, 1969; Smith et al., 1998; Burke et al., 2001; Lafreniere-Roula and McCrea, 2005; Ivanenko et al., 2006; Rybak et al., 2006a,b). Recent studies of locomotion facilitated by epidural stimulation have provided some new perspectives on how specific locomotor networks in rats, cats, and humans func-

\footnotetext{
Received Jan. 8, 2008; revised April 28, 2008; accepted May 2, 2008.

This work was supported by National Institutes of Health Grant NS16333, Russian Foundation for Basic Research -U.S. Civilian Research and Development Foundation Grant 07-04-91106, and the Christopher Reeve Paralysis Foundation (Spinal Cord Consortium, Springfield, NJ). We thank Sharon Zdunowski for help with technical assistance and constructive comments and Maynor Herrera for excellent animal care.

The authors declare no competing financial interests.

Correspondence should be addressed to Dr. V. Reggie Edgerton, Department of Physiological Science, University of California, Los Angeles, 621 Charles E. Young Drive LS 1804, Los Angeles, CA 90095-1527. E-mail: vre@ucla.edu. D01:10.1523/JNEUROSCI.0080-08.2008

Copyright $\odot 2008$ Society for Neuroscience $\quad 0270-6474 / 08 / 286022-08 \$ 15.00 / 0$
}

tion in vivo (Dimitrijevic et al., 1998; Dimitrijevic and Dimitrijevic, 2002; Gerasimenko et al., 2003, 2006, 2007).

We have observed that an electrical shock at the $S 1$ spinal cord segment evokes a direct early response, a monosynaptic (middle) response, and a long-latency (late) response in both intact (Gerasimenko et al., 2006) and spinal (Lavrov et al., 2006) rats when standing bipedally. We also observed modulation of these potentials in a locomotor phase-dependent manner during bipedal stepping in intact rats (Gerasimenko et al., 2006) and in nondisabled humans (Courtine et al., 2007), demonstrating the highly predictable and dynamic nature of these evoked responses. Additional preliminary evidence of the functional significance of these evoked responses during bipedal standing was indicated by the observation that epidural stimulation evoked late responses that were absent immediately after a complete spinal cord transection but reappeared within several weeks after injury, at approximately the time that stepping began to reoccur (Lavrov et al., 2006). More recently, Gerasimenko et al. (2007) described how individual stimuli of a tonic train at $40 \mathrm{~Hz}$ applied at the S1 spinal segment selectively enhances the amplitude of the middle response during the extensor EMG burst compared with minimal modulation of this response in the flexor muscles during bipedal stepping in complete spinal rats. These experiments also demonstrated qualitative and quantitative differences between the EMG bursts in a flexor versus an extensor muscle and in the hindlimb 
kinematics in response to epidural stimulation at different speeds and at different times after a complete midthoracic spinal cord transection.

In the present study, we have examined the phase-dependent modulation of the middle and late responses to epidural stimulation in complete spinal rats during bipedal stepping. Furthermore, we have characterized how this phase-dependent modulation changes over time after a spinal cord injury, as well as how this modulation relates to stepping ability. We observed a robust and highly predictable gating mechanism that modulates evoked responses to a given motor pool in proportion to the level of excitability of that motor pool. Indeed, there was a systematic modulation of the middle and late responses that was not only phase dependent, but also a function of the level of recovery of stepping after a spinal cord injury.

\section{Materials and Methods}

Data were obtained from nine adult female Sprague Dawley rats (270$300 \mathrm{~g}$ body weight). The experimental procedures comply with the National Institutes of Health Guide for the Care and Use of Laboratory Animals and were conducted in accordance with experimental protocols approved by the Animal Care Committee at the University of California, Los Angeles.

Surgical procedures. The rats were anesthetized deeply using a combination of ketamine $(100 \mathrm{mg} / \mathrm{kg})$ and xylazine $(10 \mathrm{mg} / \mathrm{kg})$ and maintained at a surgical level with supplemental doses of ketamine as needed. All surgeries were performed under aseptic conditions.

Headplug implant. A small incision was made at the midline of the skull. The muscles and fascia were retracted laterally, small grooves were made in the skull with a scalpel, and the skull was thoroughly dried. Two 12-pin amphenol headplugs with Teflon-coated stainless-steel wires (AS632; Cooner Wire) were securely attached to the skull with screws and dental cement as described previously (Roy et al., 1991). A small skin incision was made in the lower lumbar region, and all wires were routed subcutaneously to this opening.

$E M G$ implants. Skin and fascial incisions were made to expose the bellies of the medial gastrocnemius (MG) and tibialis anterior (TA) muscles bilaterally. Two wires were routed subcutaneously from the back incision to each of the muscles. A small notch $(\sim 1 \mathrm{~mm})$ of insulation was removed from each wire to form recording electrodes, and the exposed regions were inserted and secured in the midbelly of the deep region of each muscle as described previously (Roy et al., 1991). Stimulation through the headplug was used to verify the proper placement of the electrodes in each muscle. The EMG wires were coiled near each implant site to provide stress relief.

Spinal cord transection and epidural stimulation electrode implants. A middorsal skin incision was made between T6 and L4, and the paravertebral muscles were retracted as needed. A partial laminectomy was performed at the T8-T9 level, and the dura mater was opened longitudinally. Lidocaine was applied locally, and the spinal cord was transected completely as described previously (Talmadge et al., 2002). Two surgeons verified the completeness of the spinal cord transection by lifting the cut ends of the spinal cord with fine forceps. A partial laminectomy then was performed to expose spinal cord segment S1 for implantation of the epidural stimulating electrodes. Wires from the back incision were tunneled subcutaneously to the exposed region of the spinal cord. A small notch $(\sim 1 \mathrm{~mm})$ of insulation was removed from one wire, and this wire was affixed to the dura at the midline at the $\mathrm{S} 1$ spinal segment with the exposed surface facing the spinal cord using 9.0 suture as described previously (Ichiyama et al., 2005). The exposed region of the wire served as the stimulating electrode. The wire was coiled in the back region to provide stress relief. The Teflon coating was stripped from the distal centimeter of the second wire, which then was inserted subcutaneously in the back region and served as a common ground.

All incision areas were irrigated liberally with warm, sterile saline and closed in layers, i.e., the investing fascia and then the skin. All closed incision sites were cleaned thoroughly with saline solution. Analgesia was provided by Buprenex $(0.5-1.0 \mathrm{mg} / \mathrm{kg}$, i.m., three times per day). The analgesics were initiated before completion of the surgery and continued for a minimum of $2 \mathrm{~d}$. The rats were allowed to fully recover from anesthesia in an incubator. The rats were housed individually, and the bladders of the spinal rats were expressed manually three times per day for the first 2 weeks after surgery and twice per day thereafter. The hindlimbs of the spinal rats were moved passively through a full range of motion once per day to maintain joint mobility.

Stimulation and recording procedures. Testing procedures began 1 week after surgery and were performed weekly for 7 weeks after spinal cord transection. All testing was performed when the rats were fully awake. The raw EMG signals were amplified and filtered (10-10,000 Hz bandpass). Stimulation was performed using a Grass S88 Stimulator (Grass Instruments) and a stimulus isolation unit (Grass SIU5; Grass Instruments). Epidural stimulation was administered as single stimuli (duration of $0.5 \mathrm{~ms}$ ). Early, middle, and late responses were evoked from selected hindlimb muscles via $\mathrm{ES}(0.2 \mathrm{~Hz})$ at $\mathrm{S} 1$ to determine the stimulus intensity-response amplitude curve. Averages of 10 responses were determined at stimulation intensities ranging from 0.5 to $10 \mathrm{~V}$ in increments of $0.5 \mathrm{~V}$. Continuous epidural stimulation at $S 1$ from 5 to $130 \mathrm{~Hz}$ and at a pulse duration of $0.2 \mathrm{~ms}$ was used to induce bipedal stepping. Middle and late responses were examined during S1 stimulation at frequencies ranging from 5 to $130 \mathrm{~Hz}$.

All analyses of the evoked responses in the MG and TA were performed on rats that showed no spontaneous rhythmic activity on the moving treadmill belt but generated bilateral stepping with epidural stimulation. During the testing of locomotor ability, the spinal rats were secured in an upper body harness support system, and the hindlimbs were placed on a moving treadmill as described previously (Ichiyama et al., 2005). The automated body weight support system was used to provide the amount of body weight support necessary to enable walking. EMG activity was collected during stepping using a custom-made LabView program. As a measure of locomotor ability, the duration of continuous stepping was recorded under optimal epidural stimulation parameters. We identified the optimum stimulation intensity for enabling stepping by gradually increasing the intensity while the hindlimbs were in contact with the moving treadmill belt. This optimal intensity then was used to test the effects of epidural stimulation at different stimulation frequencies ranging from 5 to $130 \mathrm{~Hz}$ on stepping performance. All electrophysiological recordings from the muscles were analyzed within a $27 \mathrm{~ms}$ period after the stimulus artifact and were divided into three windows based on the onset latencies, i.e., from 1.5 to $6.5 \mathrm{~ms}$ for the early response, from 6.5 to $9.5 \mathrm{~ms}$ for the middle response, and from 9.5 to $27 \mathrm{~ms}$ for the late response (Gerasimenko et al., 2006). The amplitude is reported as the mean of 10 maximum peak-to-peak intervals.

A four-camera system with retro-reflective markers placed on bony landmarks at the iliac crest, greater trochanter, lateral condyle of the femur, lateral malleolus, and the distal end of the fifth metatarsal on both legs was used to record the kinematics of the hip, knee, and ankle joints. Video analysis of the kinematics of the stepping movements was performed using a SIMI Motion Analysis System and was used to produce stick diagrams and trajectories of the limb movements.

Statistical analyses. All data are reported as mean \pm SEM. Statistically significant differences were determined using a one-way repeatedmeasures ANOVA. Normality of the data was evaluated using the Kolmogorov-Smirnov test. Values that were not normally distributed were analyzed using the nonparametric Kruskal-Wallis rank test. The criterion level for determination of statistical significance was set at $p<0.05$ for all comparisons.

\section{Results \\ Epidural stimulation-induced responses in the hindlimb muscles}

Epidural stimulation produced three types of responses as recorded by EMG signals from selected hindlimb muscles. While in a bipedal standing position, a single electrical shock at S1 evoked early, middle, and late responses as described previously in nondisabled (Gerasimenko et al., 2006) and spinal (Lavrov et al., 

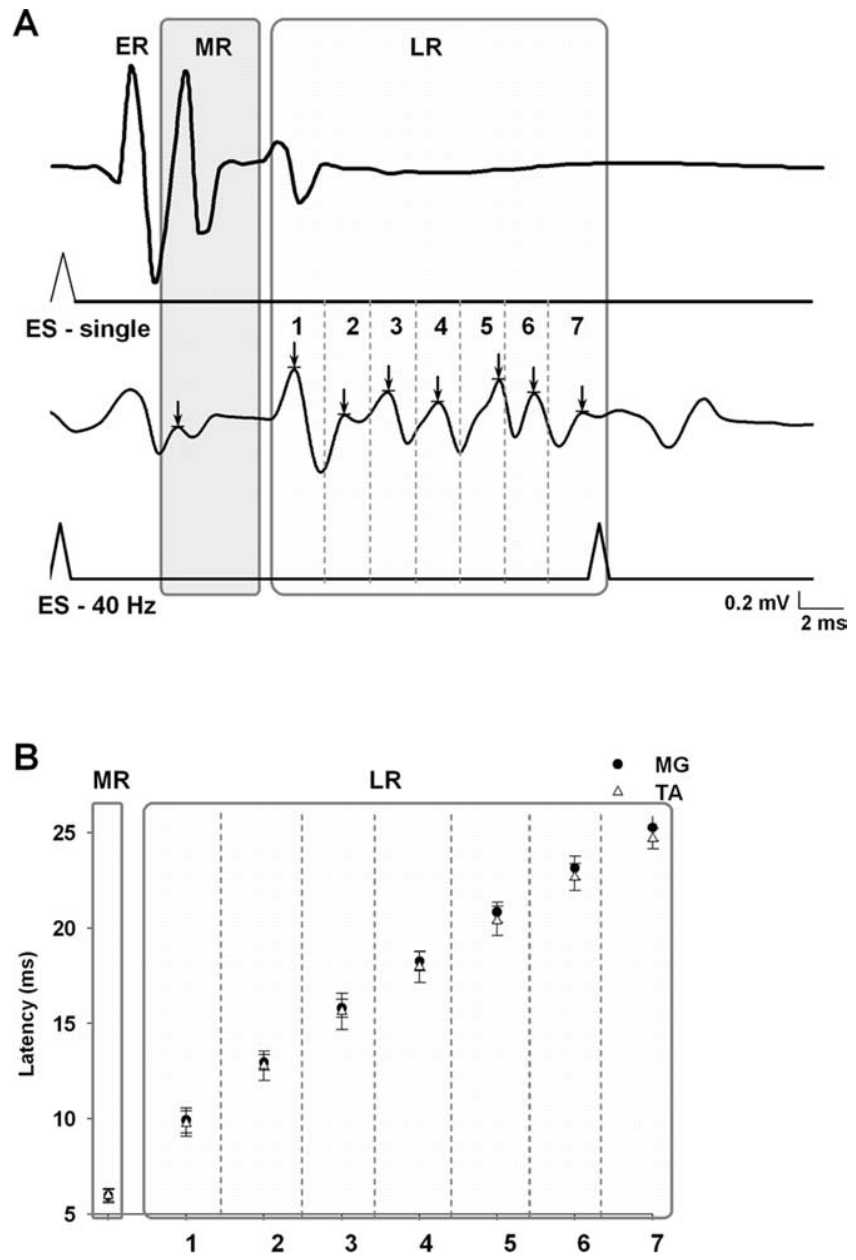

Figure 1. A, Example of spinal cord motor evoked responses to $E S$ at $S 1$ in the $M G$ muscle in a spinal cord-transected rat during standing [single shock (ES-single); top traces] compared with stepping [ $40 \mathrm{~Hz}$ stimulation (ES-40 Hz); bottom traces] on a treadmill at $7 \mathrm{~cm} / \mathrm{s}$. The potentials marked as ER (early response) have been shown to be directly evoked potentials that become prominent at higher stimulation voltages, such as that used for the single shock during standing (Lavrov et al., 2006; Gerasimenko et al., 2007). The potentials marked as MR (middle response) have the characteristics of a monosynaptic response. The potentials marked as $L R$ (late response) show one peak in response to a single shock and a sequence of peaks (labeled 1-7) during $40 \mathrm{~Hz}$ stimulation. $B$, Latency of the MR and LR in the MG and TA muscles during stepping on a treadmill. The number of peaks (1-7; arrows) in the LR in $\boldsymbol{A}$ corresponds to the latency for each peak of the $L R$ in $\boldsymbol{B}$. The arrow in the shaded region marks the MR response. The mean latency of each time bin was significantly greater than the previous time bin. These delays were not significantly different between the TA and MG. The means \pm SEM for each of the seven time bins were derived from evoked potentials of 10 bursts from nine rats.

2006) animals. The same three responses were observed during bipedal stepping on a moving treadmill belt facilitated by epidural stimulation $(40 \mathrm{~Hz})$ at $\mathrm{S} 1$. During stepping, however, the late response appeared as a long-latency complex with between five and seven spikes, in contrast to the single spike observed when epidural stimulation was delivered with the rat in a standing position (Fig. $1 A$ ).

The latency and features of the late response observed during standing suggest that this response may be attributable to interneuronal networks (Gerasimenko et al., 2006). We have reported that this response disappears immediately after a complete spinal cord transection and then reappears while standing in concert with the restoration of the ability to step when facilitated by epidural stimulation (Lavrov et al., 2006). During stepping, however, the late response is more complex in that it includes several

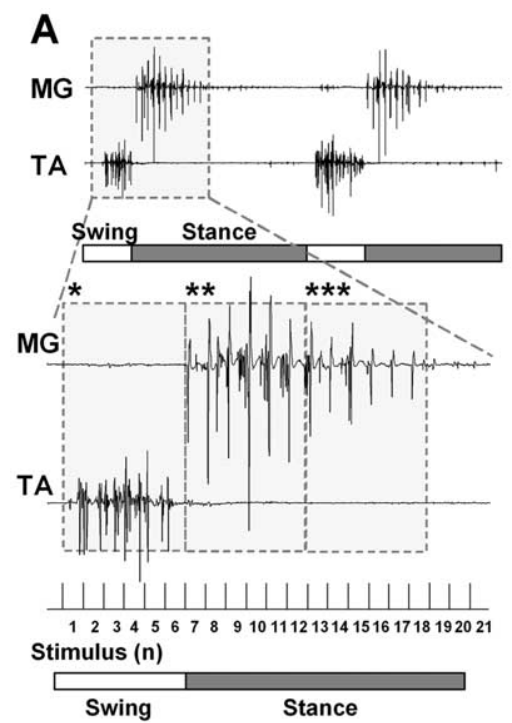

B

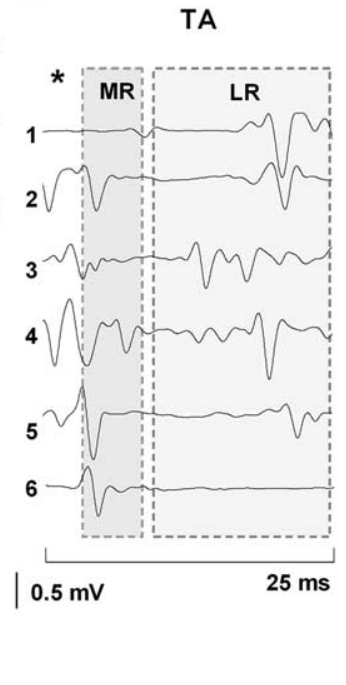

C

MG
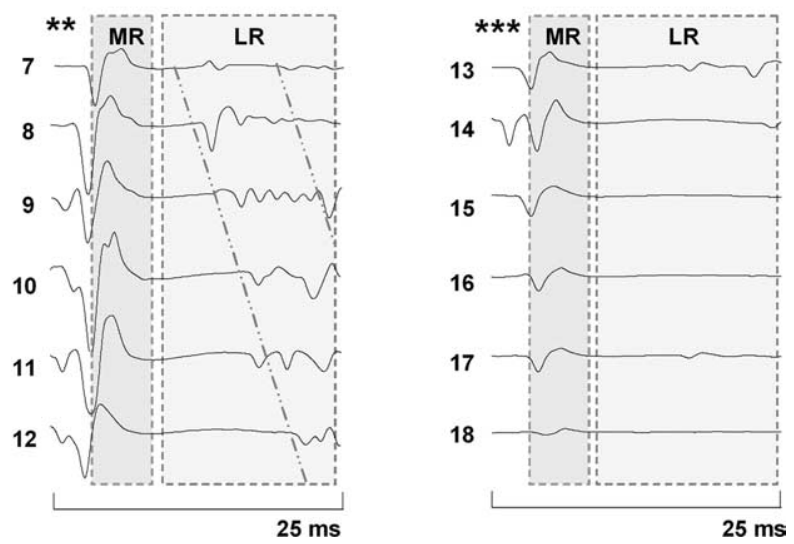

Figure 2. $A$, Formation of EMG bursts in the MG and TA muscles from the MR and LR during epidural stimulation-induced $(40 \mathrm{~Hz})$ stepping in a spinal rat. $\boldsymbol{B}, \boldsymbol{C}$, Induction of a series of MRs and LRs in the TA and MG muscles, respectively, during one step cycle. Asterisks in $\boldsymbol{B}$ and $\boldsymbol{C}$ correspond to the interval noted with the same number of asterisks in $A$. Horizontal bars: open, swing phase; filled, stance phase of a step cycle. The diagonal lines in Chighlight the shift in the latency of the LR in the MG with consecutive stimuli. Abbreviations are the same as in Figure 1.

spikes. The consistency in the number of spikes and in their latencies is illustrated on Figure $1 B$. Because these complex responses were observed during stepping on a treadmill but not during quiet standing (Fig. $1 A$ ), it is presumed that they reflect activation of spinal circuits (a state-dependent feature) that contribute to locomotor performance.

\section{Spinal cord short- and long-latency responses and the} formation of rhythmic EMG activity during epidural stimulation

Analysis of the EMG activity in hindlimb muscles during stepping on a treadmill facilitated by epidural stimulation $(40 \mathrm{~Hz}) 5$ weeks after a complete spinal cord transection showed that both middle and late responses participated in the formation of rhythmic EMG bursting (Fig. 2). MG EMG burst formation during epidural stimulation usually began with the appearance of a middle response with a latency of 5-7 ms (Fig. 2C, stimulus 7). After the middle response, a late response usually appeared with a progressive delay between each stimulus (Fig. 2C). As the EMG burst amplitude declined (Fig. $2 \mathrm{~A}$ ), there was a concomitant decrease 
in the amplitude and number of late response spikes (Fig. 2C). Only the middle response was present during the last portion of the EMG burst. In turn, the middle response was completely inhibited at the termination of the burst and reappeared at the initiation of the next EMG burst.

In contrast with the MG EMG burst, the TA included both the middle and late responses during the entire duration of the EMG burst (Fig. 2B). The recovery of the late response after spinal cord transection was observed earlier and the amplitude was higher in the TA than in the MG, whereas the middle response recovered within a similar time frame and with a similar amplitude in the MG and TA muscles (Fig. 3). Formation of the motor pattern for stepping based on the middle and late response components in the MG and TA muscles strongly depended on the phase of the step cycle. The late response occurred during the swing phase in the TA and the first part of the stance phase in the MG, whereas only a middle response occurred in the MG during the last portion of the stance phase (Figs. 2, 3).

A shift in the latencies of the longlatency components of the late response was observed in the MG EMG burst (Fig. $2 C)$. During the first half of the EMG burst, the late response is comprised primarily of peaks with latencies between 14.4 and 16.2 ms (Fig. $4 B$ ). During the second half of the MG EMG burst, the long-latency response latencies increase to between 18.9 and 22.8 ms. In contrast, these latencies in the TA muscle remain unchanged throughout the EMG burst, i.e., between 17.0 and $18.4 \mathrm{~ms}$ (Fig. 4A).

\section{Effects of frequency of epidural} stimulation on facilitation of stepping We studied the effects of stimulation frequencies ranging from 30 to $130 \mathrm{~Hz}$ in facilitating stepping in spinal rats (Fig. 5). The most effective frequencies were between 40 and $60 \mathrm{~Hz}$ (Fig. 5A, $50 \mathrm{~Hz}$ ). Epidural stimulation at low frequencies $(<40$ $\mathrm{Hz}$ ) initially evoked a low-amplitude, poorly modulated rhythmic pattern that usually required a considerable amount of time to establish a reciprocal rhythmic pattern between flexor and extensor muscles (Fig. 5A, $30 \mathrm{~Hz}$ ). At higher frequencies $(>60 \mathrm{~Hz})$, epidural stimulation produced high-amplitude, irregular patterns of activity (Fig. 5A, 90 and 130 $\mathrm{Hz}$ ). In fact, during the first few seconds after beginning epidural stimulation at frequencies $>60 \mathrm{~Hz}$, there was a temporary, highamplitude irregular tonic activity that subsequently became a rhythmic, irregular pattern.

After termination of epidural stimulation (stimulus off), successful stepping was continued for the longest period at frequencies between 40 and $60 \mathrm{~Hz}$ (Fig. $5 \mathrm{C}, 50 \mathrm{~Hz}$ ), whereas
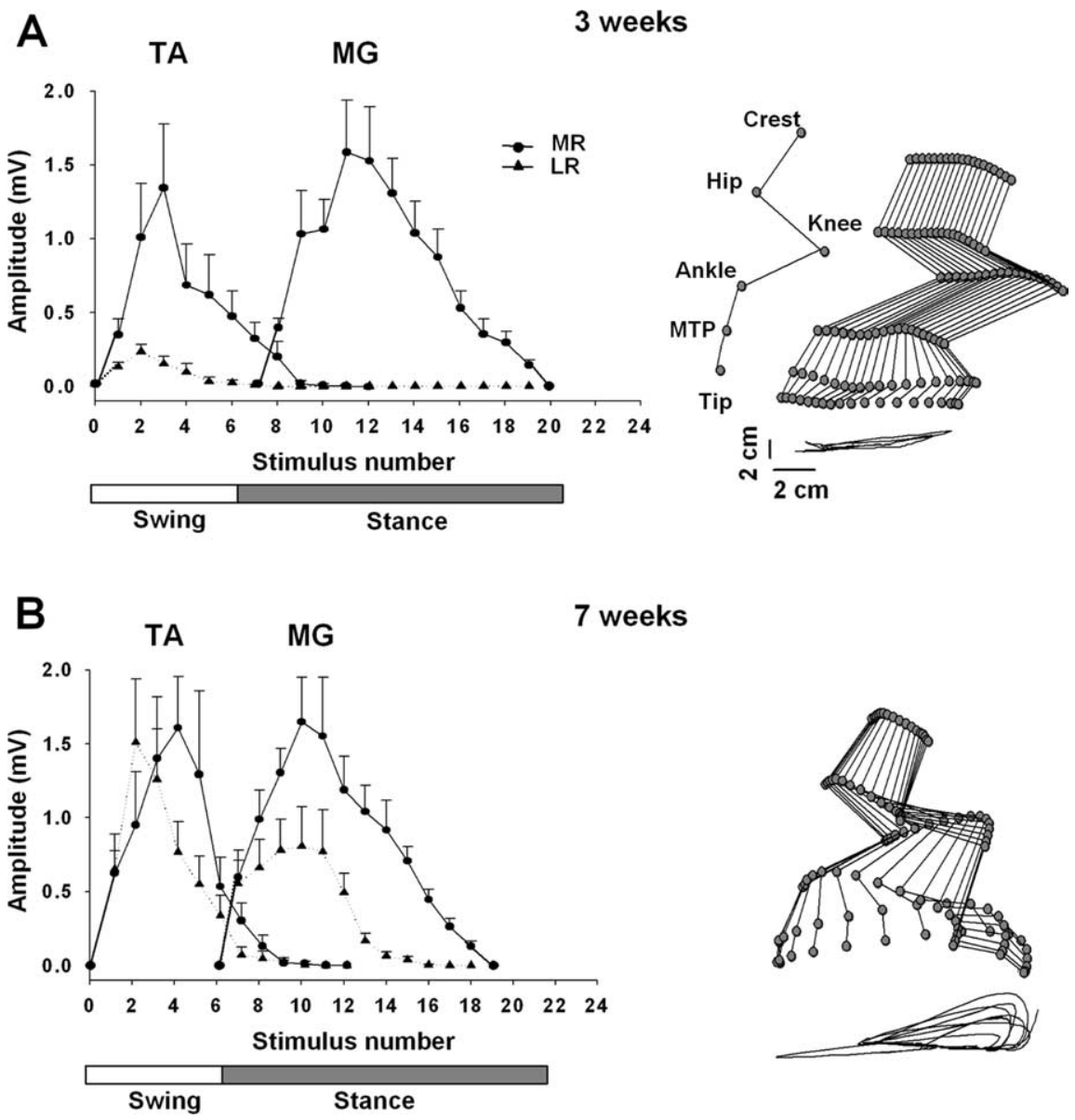

7 weeks

Figure 3. $A, B$, Modulation of the MR and LR during stepping on a treadmill at $7 \mathrm{~cm} / \mathrm{s}$ facilitated by $\mathrm{ES}$ at $\mathrm{S} 1(40 \mathrm{~Hz})$ at 3 and 7 weeks after spinal cord transection, respectively. Values are mean \pm SEM for nine rats. Note the substantial recovery of the LR at 7 weeks compared with 3 weeks after spinal cord transection. Representative stick diagrams of the hindlimb movements during the swing phase at 3 and 7 weeks after spinal cord transection are shown on the right. The time between individual sticks is $30 \mathrm{~ms}$. The trajectories of the ankle for seven consecutive steps are shown at the bottom of each stick figure. Joint angles are identified in $\boldsymbol{A}$. Abbreviations are the same as in Figure 1 .
A

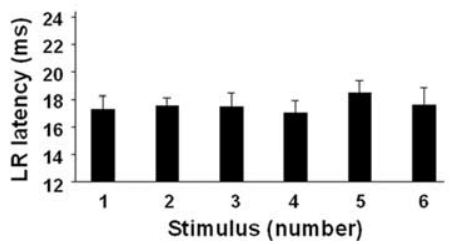

B

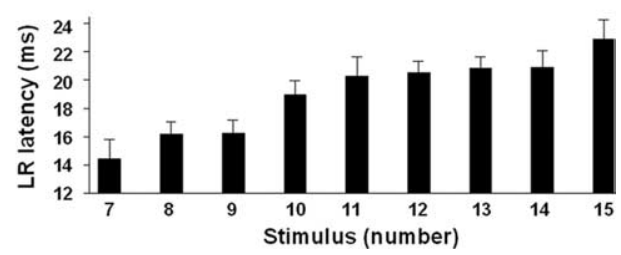

Figure 4. $\quad \boldsymbol{A}, \boldsymbol{B}$, Mean \pm SEM latencies of the maximal $L R$ for the TA and $M G$ muscles for the spinal rats shown in Figure 2. The numbers on the abscissas (1-15) correspond to the sequence of stimuli in one step cycle as shown in Figure $2 A$. The latencies of the LR for a sequence of six stimuli during the TA burst remained relatively constant, whereas the latencies for a series of similar responses in the MG increased progressively. Abbreviations are the same as in Figure 1.

the stepping pattern rapidly became unstable and the EMG activity nonreciprocal at lower (Fig. $5 \mathrm{C}, 30 \mathrm{~Hz}$ ) or higher (Fig. $5 C, 90$ and $130 \mathrm{~Hz}$ ) frequencies. In addition, the step cycle period was significantly less variable at $50 \mathrm{~Hz}$ than at lower and higher frequencies of epidural stimulation (Fig. 6). We then compared the middle and late responses in the MG at different frequencies of epidural stimulation with those at $30 \mathrm{~Hz}$ (interpulse interval of $33.3 \mathrm{~ms}$ ) (Fig. 5B). The first stimulus of a train produced only a middle response at $30 \mathrm{~Hz}$ stimulation, but both a middle and late response at $50 \mathrm{~Hz}$ stimulation. At $30 \mathrm{~Hz}$ 
A

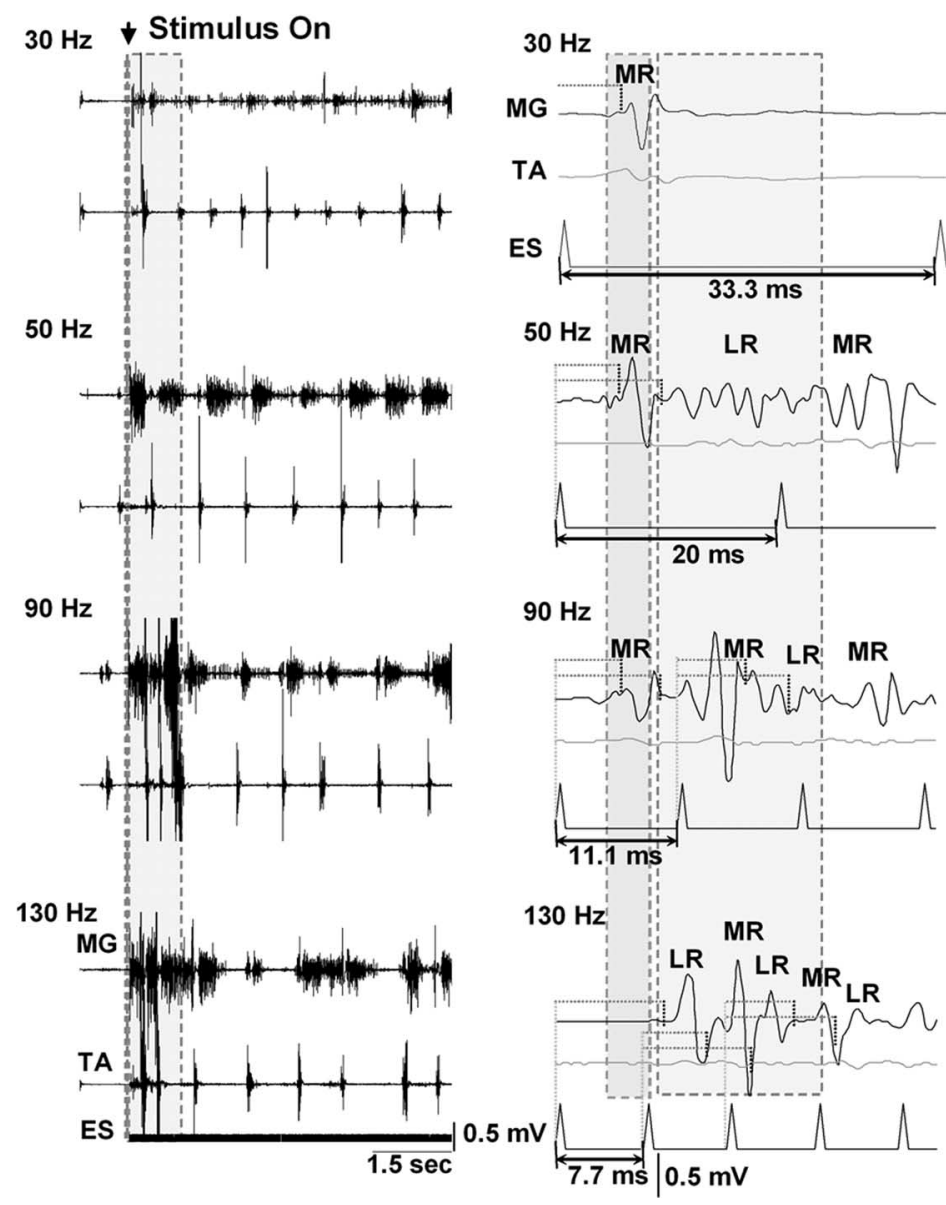

C

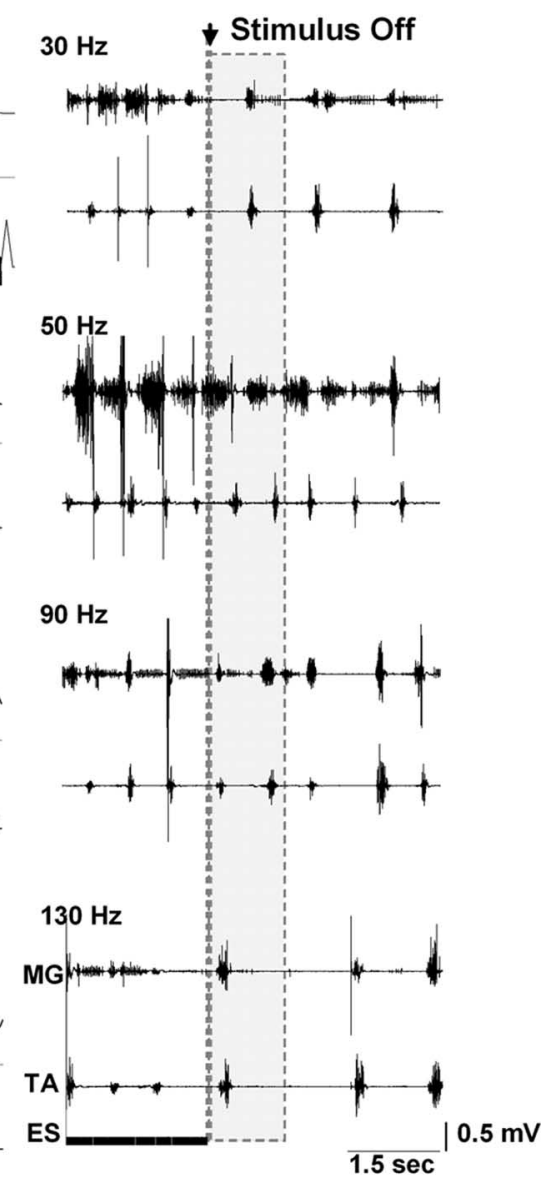

Figure 5. $A$, Effect of different frequencies of ES on the initiation of stepping in spinal rats. Shaded areas in $A$ represent the initial period after the onset of ES: note the transitional phase involving disorganized, high-amplitude activity at the higher $(90$ and $130 \mathrm{~Hz})$, but not the lower $(30$ and $50 \mathrm{~Hz})$, stimulation frequencies. The most consistent bursting pattern is seen at $50 \mathrm{~Hz}$. $\boldsymbol{B}$, Note the different modulation of the MR and LR at different frequencies of stimulation. The first shaded area shows the MR from the first stimulus. The most consistent stepping at $50 \mathrm{~Hz}$ in $\boldsymbol{A}$ corresponds to the appearance of an LR between the MRs of the first and second stimuli (second shaded area). At higher frequencies, the MRs of subsequent stimuli overlap the LRs. The horizontal dotted lines identify the latencies for the MR and LR. C, Comparisons of the bursting patterns at different frequencies when the epidural stimulation is terminated. Note that the most robust bursting and alternating pattern immediately after the stimulus is turned off is observed at $50 \mathrm{~Hz}$ (shaded area). Abbreviations are the same as in Figure 1.

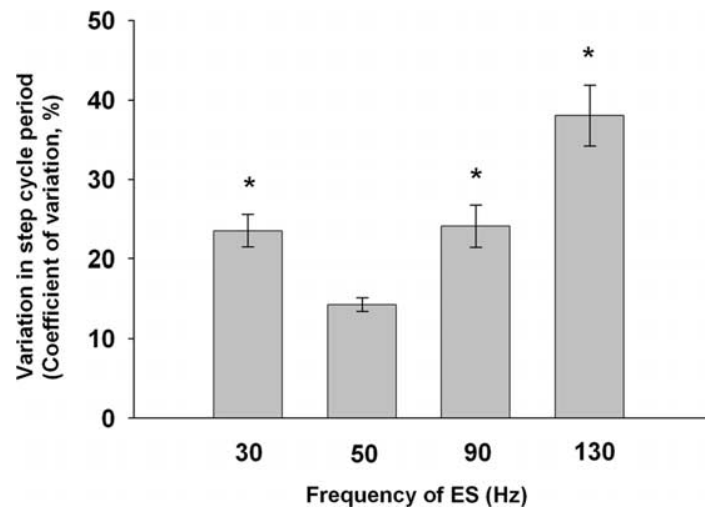

Figure 6. Variability in the step cycle period at different frequencies of ES. The mean \pm SEM variability was significantly higher at all other frequencies than at $50 \mathrm{~Hz}\left(n=6\right.$ rats; ${ }^{*} p<$ 0.001 , Kruskal-Wallis rank test).

stimulation, a late response was observed only after several consecutive stimuli (data not shown). At frequencies $>60 \mathrm{~Hz}$, there was an immediate overlap between the late response and the subsequent middle response.
Recovery of motor-evoked responses in the hindlimb muscles after a complete spinal cord transection

Epidural stimulation-induced stepping performance improved with time after spinal cord transection, as reflected in the EMG bursting patterns of the MG and TA muscles (Fig. 7A). Coordinated, alternating bursting between the extensor and flexor muscles was observed initially at $\sim 5$ weeks after spinal cord transection. Primarily short-latency monosynaptic responses were observed in the MG at 2 and 3 weeks after spinal cord transection during epidural stimulation-facilitated stepping, whereas both short-latency and long-latency responses were present by 5 weeks after transection (Fig. 7B). Thus, improved stepping ability was coincident with the reappearance of long-latency responses.

The middle and late responses during epidural stimulation induced stepping were modulated differently in the MG and TA muscles between 3 (Fig. $3 A$ ) and 7 (Fig. 3B) weeks after spinal cord transection. At 3 weeks after transection, only a middle response was observed in the MG, whereas both a middle and late response were present in the TA (Fig. 3A). In addition, the amplitude of the middle response was much higher than that of the late response in the TA. At 7 weeks after spinal cord transection, a middle response was still robust in both muscles and of an am- 

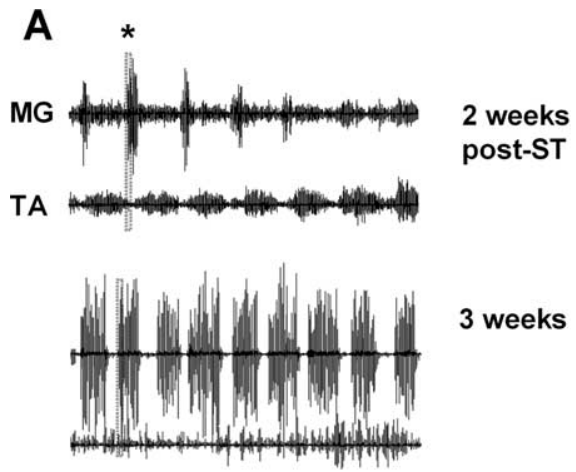

3 weeks

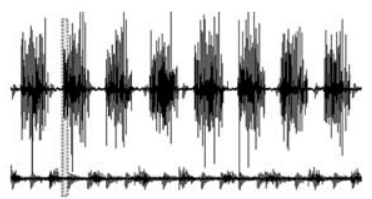

5 weeks

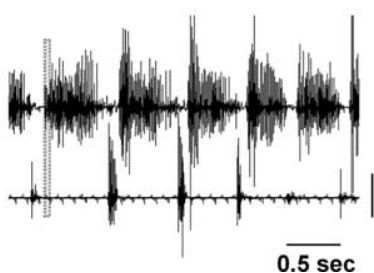

7 weeks
B

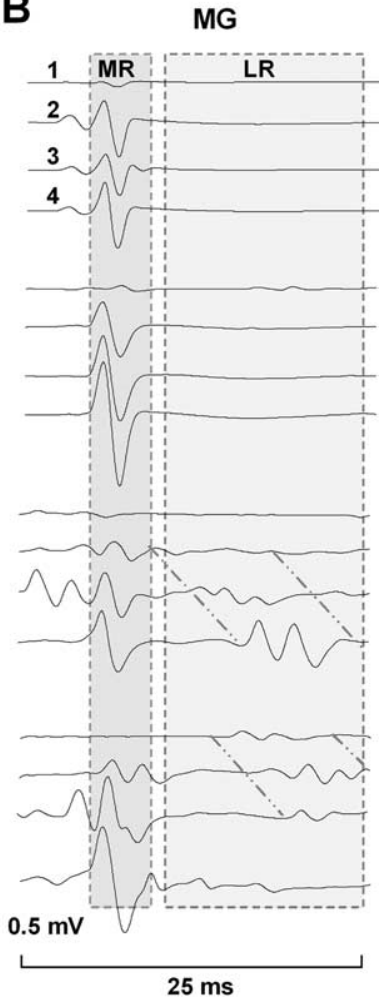

Figure 7. A, EMG bursting patterns for the MG and TA of a representative spinal rat during treadmill stepping at $7 \mathrm{~cm} / \mathrm{s}$ facilitated by ES at $\mathrm{S} 1(40 \mathrm{~Hz})$ 2, 3, 5, and 7 weeks after spinal cord transection. $\boldsymbol{B}$, MR and LR in the MG during four consecutive stimuli at the same points as in $\boldsymbol{A}$. The shaded areas ( $25 \mathrm{~ms}$ ) in the EMG recordings at each time point in $\boldsymbol{A}$ are expanded in $\boldsymbol{B}$. Note the absence of an $L$ at 2 and 3 weeks and the reappearance at 5 weeks after spinal cord transection. Note also the increasing delay in the LR with consecutive stimuli highlighted by diagonal lines in $\boldsymbol{B}$. Abbreviations are the same as in Figure 1.

Why is frequency of epidural stimulation an important factor in facilitating locomotion?

The frequency requirement for spinal cord stimulation to produce stepping is variable among species and types of stimulation (Gerasimenko et al., 2008). In decerebrated cats, epidural stimulation produces stable stepping at $3-10 \mathrm{~Hz}$ (Iwahara et al., 1991; Gerasimenko et al., 2003), whereas intraspinal stimulation produces stepping at $40-60 \mathrm{~Hz}$ (Kazennikov and Shik, 1988). Intraspinal microstimulation in cats spinalized 5-7 $\mathrm{d}$ before testing produces stepping at 2-6 Hz (Barthélemy et al., 2007). The optimum frequency for initiation of rhythmic movements with epidural stimulation in human is between 30 and $40 \mathrm{~Hz}$ (Dimitrijevic et al., 1998; Minassian et al., 2007) and is correlated with the appearance of long-latency components in the EMG bursts (Jilge et al., 2004; Minassian et al., 2004). Although this variability in the optimum stimulation frequency for facilitating or inducing stepping can be attributed to multiple factors, our observations suggest that the specific spinal circuits activated determine the optimal frequency. EMG responses at different frequencies indicate that the optimal stimulation frequencies $(40-60 \mathrm{~Hz})$ correspond to an interpulse interval that allows the entire late response to occur uninterrupted by subsequent stimuli. Stimulation at $>60 \mathrm{~Hz}$ results in a middle response that interferes

plitude similar to that observed at 3 weeks after transection (Fig. $3 B$ ). The amplitude of the late response in the TA, however, was greater at 7 than at 3 weeks after spinal cord transection and of an amplitude similar to the middle response. In addition, a late response was observed in the MG at 7 weeks after transection, although only approximately one-half the amplitude of the middle response. This modulation in the evoked motor potentials corresponded to graded improvements of epidural stimulation-facilitated stepping performance. At 3 weeks after transection, stepping consisted of a stance phase on the dorsal aspect of the foot and/or with the toes curled while the paw dragged extensively along the treadmill belt during the swing phase (Fig. 3A). By 7 weeks after transection, the animals stepped with plantar placement during the stance phase and showed minimal dragging of the paw during the swing phase (Fig. $3 B)$.

\section{Discussion}

We recently demonstrated progressive postlesion changes in the middle and late responses in flexor and extensor muscles in spinal rats in a standing position (Lavrov et al., 2006) and their phasedependent modulation during stepping in normal rats (Gerasimenko et al., 2006) and humans (Courtine et al., 2007). The present data demonstrate that phase-dependent modulation observed in control rats and humans is also present in complete spinal rats after a critical level of recovery of locomotor ability. These results show a close relationship between the time of emergence of phase-dependent modulation of late responses and the point at which stepping ability recovers. with the generation of a subsequent late response (Fig. $4 B$ ). Stimulation at $<40 \mathrm{~Hz}$ was insufficient to activate the spinal network components critical for sustaining locomotion, demonstrated by the absence of a stimulation-elicited late response. The functional significance of stimulation frequency is demonstrated further by the fact that after termination of stimulation at frequencies $>60$ or $<40 \mathrm{~Hz}$, sustained rhythmic stepping occurred for shorter periods than after stimulation between 40 and $60 \mathrm{~Hz}$.

\section{Phase-dependent modulation of motor-evoked potentials}

Recovery of stepping as facilitated by epidural stimulation was coincident with a strong phase-dependent modulation of both short- and long-latency motor-evoked responses, reflected in the progressive shift in the delays of long-latency responses during the extensor, but not flexor, EMG bursts. Presynaptic mechanisms acting on muscle spindle afferents were probably involved in the phase-dependent modulation of the monosynaptic middle response during stepping, as reported in cats (Gorassini et al., 1994) and humans (Courtine et al., 2007). Ib and II proprioceptive afferents also likely played a role in determining the duration, frequency, timing, and amplitude of EMG bursts during stepping (Donelan and Pearson, 2004). It seems reasonable that modulation of long-latency responses was mediated by interneurons that influence, or control, the net excitability of flexor and extensor motor pools via skin afferents (Zehr and Stein, 1999; ValeroCabre et al., 2004; Lavrov et al., 2006). A striking feature of the evoked potentials in the TA and MG was the strong phase dependence of the amplitude of these potentials. Similar observations 
have been made in uninjured rats (Gerasimenko et al., 2006) and humans (Courtine et al., 2007) during locomotion (Edgerton et al., 2008). The present results clearly demonstrate that phasedependent gating of motor-evoked responses can be mediated by the spinal locomotor circuitry. These data are remarkably similar to the phase-dependent cutaneous reflex reversal observed when spinal cats are tripped (Forssberg et al., 1980).

\section{What are the middle and late responses evoked by epidural stimulation?}

Our previous findings suggest that the middle response corresponds to spinal cord monosynaptic circuits (Lavrov et al., 2006), and its latency is similar to the response recorded with magnetic spinal cord stimulation in normal and spinal rats (Chiba et al., 2003). The latency of the first component of the late response recorded during standing corresponds to more then one synaptic delay (Lavrov et al., 2006), and epidural stimulation and afferent input from a moving treadmill facilitate the multiple spikes in late response, when each successive spike occurs at a latency of $\sim 2$ $\mathrm{ms}$. We speculate that the multiple potentials of the late response reflect primarily variable numbers of synaptic events within the networks involved at any given phase of the step cycle. Other factors, such as repetitive firing of motoneurons that become more prominent after spinal cord injury (Harvey et al., 2006), also could contribute to these multiple components. Given that the late response is a composite response from a motor pool and therefore multiple motor units, it seems less likely that a regular interval between potentials would occur, given the relatively random nature of spike generation among motor units within a pool. Also, the intervals between the components of the late response are $\sim 2 \mathrm{~ms}$, whereas a typical interval for action potentials within a single motor unit has been reported to be $\sim 200 \mathrm{~ms}$ (Harvey et al., 2006). Other factors that could contribute to multiple potentials of late response may be the variation that must be inherent in the conduction velocities among the different afferents.

We suggest that there could be different strengths of inhibitory connections between sequential polysynaptic circuits. The networks responsible for activation of the MG motor pool could be organized such that each successive level inhibits the previous one, resulting in the observed progressive shift in the late responses. The number of interneurons of the longest circuit in this model would determine the late-response duration. Inhibitory circuits modulating the TA motor pool may be insufficient or absent, resulting in no shifting of the late response. An alternative explanation could be a reduction in membrane excitability per stimulus, in which each subsequent potential occurs with a slightly longer delay. The longer time course over which this phenomenon occurs versus the typical delay of the late response components, however, suggests that this is not a dominating factor.

There being different mechanisms underlying the middle and late responses is consistent with the complete inhibition of the late response at $3 \mathrm{~Hz}$ stimulation but only partial inhibition of the middle response even at 5-10 Hz (Skinner et al., 1996; Reese et al., 2006; Lavrov et al., 2006). The frequency-dependent depression of the middle response is similar to that observed for the H-reflex when tested acutely with sciatic nerve stimulation, suggesting a similar monosynaptic origin for both responses (Wolpaw and Chen, 2001; Lavrov et al., 2006). The different time course for the restoration of the middle and late responses after transection also suggests a different origin for these two responses. The middle response and H-reflex are restored early (Thompson et al., 1993; Valero-Cabre et al., 2004) and show facilitation within 1-2 weeks after transection (Skinner et al., 1996; Valero-Cabre et al., 2004; Reese et al., 2006; Lavrov et al., 2006). In contrast, the late response shows a gradual recovery beginning 2-3 weeks after transection with a time course similar to the recovery of the polysynaptic components of flexor-extensor reflexes tested acutely after transection (Valero-Cabre et al., 2004).

A clear difference between the evoked potentials in TA and MG was the timing of the recovery of the middle and late responses after transection. Within a few days, the middle response recovered in both muscles, whereas after 3 weeks the late response recovered somewhat in the TA but not MG. After 7 weeks, the late response amplitude increased by sevenfold in the TA, whereas in the MG it recovered from not being evident at 3 weeks to one-half the amplitude of the TA at 7 weeks. The evoked potentials forming the late response differed in the TA and MG: the progression of the onset delays during consecutive stimuli was evident only in MG. Similarly, long-latency responses were more prominent in flexors than extensors during stimulation-induced stepping in humans (Gerasimenko et al., 2001, 2008; Minassian et al., 2004) and during stimulation- and quipazine-facilitated stepping in spinal rats (Gerasimenko et al., 2007). The timing of recovery of weight-bearing stepping highlights the functional significance of the phase-dependent modulation of the late response in TA and MG. As suggested previously (Lavrov et al., 2006), the presence and amplitude of the late, but not middle, response appear to be related to the degree of locomotor recovery after transection.

The implication of the time-related robust modulation of the late response after transection and its systematic, sequential delays as a function of the step cycle phase is that these mechanisms must become viable for locomotor recovery. The challenge is in determining which motoneuronal or interneuronal properties within the lumbosacral spinal cord are playing a critical role in locomotor recovery. Identification of the dynamic nature of these mechanisms and their pharmacology under in vivo conditions and without anesthetics may provide novel therapeutic approaches to facilitate stepping after a spinal injury or other debilitating neuromotor disorders.

\section{References}

Barbeau H, Rossignol S (1991) Initiation and modulation of the locomotor pattern in the adult chronic spinal cat by noradrenergic, serotonergic and dopaminergic drugs. Brain Res 546:250-260.

Barthélemy D, Leblond H, Rossignol S (2007) Characteristics and mechanisms of locomotion induced by intraspinal microstimulation and dorsal root stimulation in spinal cats. J Neurophysiol 97:1986-2000.

Burke RE, Degtyarenko AM, Simon ES (2001) Patterns of locomotor drive to motoneurons and last-order interneurons: clues to the structure of the CPG. J Neurophysiol 86:447-462.

Chau C, Barbeau H, Rossignol S (1998) Effects of intrathecal alphal-and alpha2-noradrenergic agonists and norepinephrine on locomotion in chronic spinal cats. J Neurophysiol 79:2941-2963.

Chiba A, Oshio K, Inase M (2003) Magnetically evoked EMGs in rats. Neurol Res 25:87-91.

Courtine G, Harkema SJ, Dy CJ, Gerasimenko YP, Dyhre-Poulsen P (2007) Modulation of multisegmental monosynaptic responses in a variety of leg muscles during walking and running in humans. J Physiol (Lond) 582:1125-1139.

Dimitrijevic MM, Dimitrijevic MR (2002) Clinical elements for the neuromuscular stimulation and functional electrical stimulation protocols in the practice of neurorehabilitation. Artif Organs 26:256-259.

Dimitrijevic MR, Gerasimenko Y, Pinter MM (1998) Evidence for a spinal central pattern generator in humans. Ann NY Acad Sci 860:360-376.

Donelan JM, Pearson KG (2004) Contribution of sensory feedback to ongoing ankle extensor activity during the stance phase of walking. Can J Physiol Pharmacol 82:589-598.

Edgerton VR, Courtine G, Gerasimenko YP, Lavrov I, Ichiyama RM, Fong AJ, 
Cai LL, Otoshi CK, Tillakaratne NJ, Burdick JW, Roy RR (2008) Training locomotor networks. Brain Res Rev 57:241-254.

Engberg I, Lundberg A (1969) An electromyographic analysis of muscular activity in the hindlimb of the cat during unrestrained locomotion. Acta Physiol Scand 75:614-630.

Forssberg H, Grillner S, Halbertsma J (1980) The locomotion of the low spinal cat. I. Coordination within a hindlimb. Acta Physiol Scand 108:269-281.

Gerasimenko Y, Daniel O, Regnaux J, Combeaud M, Bussel B (2001) Mechanisms of locomotor activity generation under epidural spinal cord stimulation. In: Sensorimotor control (Dengler R, Kossev A, eds), pp 164171. Washington, DC: IOS.

Gerasimenko YP, Avelev VD, Nikitin OA, Lavrov IA (2003) Initiation of locomotor activity in spinal cats by epidural stimulation of the spinal cord. Neurosci Behav Physiol 33:247-254.

Gerasimenko YP, Lavrov IA, Courtine G, Ichiyama RM, Dy CJ, Zhong H, Roy RR, Edgerton VR (2006) Spinal cord reflexes induced by epidural spinal cord stimulation in normal awake rats. J Neurosci Methods 157:253-263.

Gerasimenko YP, Ichiyama RM, Lavrov IA, Courtine G, Cai L, Zhong H, Roy RR, Edgerton VR (2007) Epidural spinal cord stimulation plus quipazine administration enable stepping in complete spinal adult rats. J Neurophysiol 98:2525-2536.

Gerasimenko Y, Roy RR, Edgerton VR (2008) Epidural stimulation: comparison of the spinal circuits that generate and control locomotion in rats, cats and humans. Exp Neurol 209:417-425.

Gorassini MA, Prochazka A, Hiebert GW, Gauthier MJ (1994) Corrective responses to loss of ground support during walking. I. Intact cats. J Neurophysiol 71:603-610.

Graham-Brown T (1911) The intrinsic factors in the act of progression in the mammal. Proc R Soc Lond B Biol Sci 84:308-319.

Harvey PJ, Li Y, Li X, Bennett DJ (2006) Persistent sodium currents and repetitive firing in motoneurons of the sacrocaudal spinal cord of adult rats. J Neurophysiol 96:1141-1157.

Ichiyama RM, Gerasimenko YP, Zhong H, Roy RR, Edgerton VR (2005) Hindlimb stepping movements in complete spinal rats induced by epidural spinal cord stimulation. Neurosci Lett 383:339-344.

Iwahara T, Atsuta Y, Garcia-Rill E, Skinner R (1991) Spinal cord stimulation-induced locomotion in the adult cat. Brain Res Bull 28:99-105.

Ivanenko YP, Poppele RE, Lacquaniti F (2006) Motor control programs and walking. Neuroscientist 12:339-348.

Jilge B, Minassian K, Rattay F, Dimitrijevic MR (2004) Frequencydependent selection of alternative spinal pathways with common periodic sensory input. Biol Cybern 91:359-376.

Kazennikov OV, Shik ML (1988) Propagation of the activity along the "stepping strip" of the spinal cord in the cat. Neirofiziologiia 20:763-769.

Lafreniere-Roula M, McCrea DA (2005) Deletions of rhythmic motoneu- ron activity during fictive locomotion and scratch provide clues to the organization of the mammalian central pattern generator. J Neurophysiol 94:1120-1132.

Lavrov I, Gerasimenko YP, Ichiyama RM, Courtine G, Zhong H, Roy RR, Edgerton VR (2006) Plasticity of spinal cord reflexes after a complete transection in adult rats: relationship to stepping ability. J Neurophysiol 96:1699-1710.

Minassian K, Jilge B, Rattay F, Pinter MM, Binder H, Gerstenbrand F, Dimitrijevic MR (2004) Stepping-like movements in humans with complete spinal cord injury induced by epidural stimulation of the lumbar cord: electromyographic study of compound muscle action potentials. Spinal Cord 42:401-416.

Minassian K, Persy I, Rattay F, Pinter MM, Kern H, Dimitrijevic MR (2007) Human lumbar cord circuitries can be activated by extrinsic tonic input to generate locomotor-like activity. Hum Mov Sci 26:275-295.

Reese NB, Skinner RD, Mitchell D, Yates C, Barnes CN, Kiser TS, Garcia-Rill E (2006) Restoration of frequency-dependent depression of the H-reflex by passive exercise in spinal rats. Spinal Cord 44:28-34.

Roy RR, Hutchison DL, Pierotti DJ, Hodgson JA, Edgerton VR (1991) EMG patterns of rat ankle extensors and flexors during treadmill locomotion and swimming. J Appl Physiol 70:2522-2529.

Rybak IA, Shevtsova NA, Lafreniere-Roula M, McCrea DA (2006a) Modeling spinal circuitry involved in locomotor pattern generation: insights from deletions during fictive locomotion. J Physiol (Lond) 577:617-639.

Rybak IA, Stecina K, Shevtsova NA, McCrea DA (2006b) Modeling spinal circuitry involved in locomotor pattern generation: insights from the effects of afferent stimulation. J Physiol (Lond) 577:641-658.

Skinner RD, Houle JD, Reese NB, Berry CL, Garcia-Rill E (1996) Effects of exercise and fetal spinal cord implants on the H-reflex in chronically spinalized adult rats. Brain Res 729:127-131.

Smith JL, Carlson-Kuhta P, Trank TV (1998) Forms of forward quadrupedal locomotion. III. A comparison of posture, hindlimb kinematics, and motor patterns for downslope and level walking. J Neurophysiol 79:1702-1716.

Talmadge RJ, Roy RR, Caiozzo VJ, Edgerton VR (2002) Mechanical properties of rat soleus after long-term spinal cord transection. J Appl Physiol 93:1487-1497.

Thompson FJ, Reier PJ, Parmer R, Lucas CC (1993) Inhibitory control of reflex excitability following contusion injury and neural tissue transplantation. Adv Neurol 59:175-184.

Valero-Cabre A, Fores J, Navarro X (2004) Reorganization of reflex responses mediated by different afferent sensory fibers after spinal cord transection. J Neurophysiol 9:2838-2848.

Wolpaw JR, Chen XY (2001) Operant conditioning of rat H-reflex: effects on mean latency and duration. Exp Brain Res 136:274-279.

Zehr EP, Stein RB (1999) What functions do reflexes serve during human locomotion? Prog Neurobiol 58:185-205. 